\title{
An Enhancement of Deep Face Technique Using Neural Network
}

\author{
Gurpreet Kaur $^{1}$ and Sukhvir Kaur ${ }^{2}$ \\ Department of CSE, I.K.G Punjab Technical University \\ ${ }^{1}$ preeti.k.dhillon@gmail.com, ${ }^{2}$ sukhsain.17@gmail.com
}

\begin{abstract}
Face recognition is an assignment that people perform routinely and easily in their everyday lives. The most recent decade has seen a pattern towards an inexorably universal nature, where compelling and minimal effort registering frameworks are, no doubt coordinated into cell telephones, autos, therapeutic instruments and very nearly every part of our lives. In the previous work, few researchers have focused on detection of face using some methodology. In this research paper, the face recognition system proposed the Detection time, false negative in missed faces and optimality of the face. This proposed research work has been focused on optimality features of the neural network for the face images and detection time. In this paper, we have applied the neural network for three parameters such - detection time, false acceptance rate, successful rates, no. of failure, and cross correlation. Our proposed parameters provide better result as compared to the previous methodology.
\end{abstract}

Keywords: Face recognition, False acceptance rate, Cross Correlation, Neural network,Feature

\section{Introduction}

Face recognition is a visual example recognition issue where a three-dimensional article is to be distinguished focused around its two-dimensional picture. as of late, huge advancement has been made around there; owing to better face displays and all the more influential workstations, face recognition framework can accomplish great comes about under compelled circumstances. However on the grounds that face pictures are affected by a few components: light, head posture, interpretation et cetera, when all is said in done conditions, face recognition is as of now testing.

Face recognition is one of most relevant applications of image analysis. Face Recognition refers to a task of analyzing an already detected face and telling exactly whose it is and also deals with unique characteristics (facial) of human beings

In facial recognition, there are two main types of comparisons such Verification and Identification. In verification system, compares the given individual and a decision is given by yes or no. In identification process, system compares the given individual to all other individuals in the database and gives a list of the matches.

The common approach helps using neural network. Neural Network is more capable of deriving multi-orientation information to various source analyses from a face image at different scenarios and scales with the derived information being of local nature from a particular source. The common approach helps using neural network.

Face recognition is to construct a filter bank for different scales and orientations to filter which has given face image with all filters from the bank storage. These approaches will provide results in an explosion of information as the dimensionality reduction of the input face pattern is growing by a factor equaling to the number of filters in the filter bank. The amount of data in neural network, face representation is commonly used to reduce information to getting detailed information and more manageable size by 
exploiting various down sampling techniques, feature selection and subspace projection analysis before it is finally applied to a classifier in many working research field.

\section{Existing Work}

In among research reviews, the few researchers are working with different methods, Yamin Taigman et. al., in 2014 have presented closing the gap to human level performance in face verification which is based on conventional pipeline. The conventional pipeline consist detection, alignment, representation and classification to face images. This pipeline methodology was used for the 3D face image to fill the gap for the better performance. Wang et. al., in 2008 proposed to inspect a correlation matrix constructing a bank of Bayesian Networks with the goal of selecting such various filter parameters used in filters comprising the filter bank would be as uncorrelated as possible. The same methodology can also be used to visualization and found difference between the classical and the principal Bayesian Networks. John Canny et. al., in 2010 have presented a novel computational approach to edge detection proposed in their paper the basics of image processing concepts

S. Mallat et. al., in 2010 has presented Singularity detection and image processing, learning in machine, networks, fractals, genetic algorithms, wavelets, and data mining for better performance. In his journal, he suggested a min-max composition rule to measure the similarity in various types of images.

J. Canny et. al., in 1998 studied on a computational approach to edge detection, and proposed the image denoising using multimodal keywords. Rishi R. et. al., in 1979 has presented to research interests including pattern recognition, regression techniques, image processing, and data mining. These are responsible for the analytics and models required to reduce the risk factor of the American Express credit card business. R. Chaudhuri et. al., in 2003 has reviewed in fields of research interest include pattern recognition, processing with wavelet and proposed the use of denoising and face detection.

Yamin Taigman et. al., in 2014 have presented closing the gap to human level performance in face verification which is based on conventional pipeline. The conventional pipeline consist detection, alignment, representation and classification to face images. This pipeline methodology was used for the 3D face image to fill the remaining difference for the accurate and best performance. The same methodology can also be used to visualization and found difference between the classical and the principal Bayesian Networks.

\section{Methodology}

In research paper, we have proposed the face recognition methodology using by neural network and PCA. The flow of process has mentioned in the following flow charts is shown in Figure no 1: 


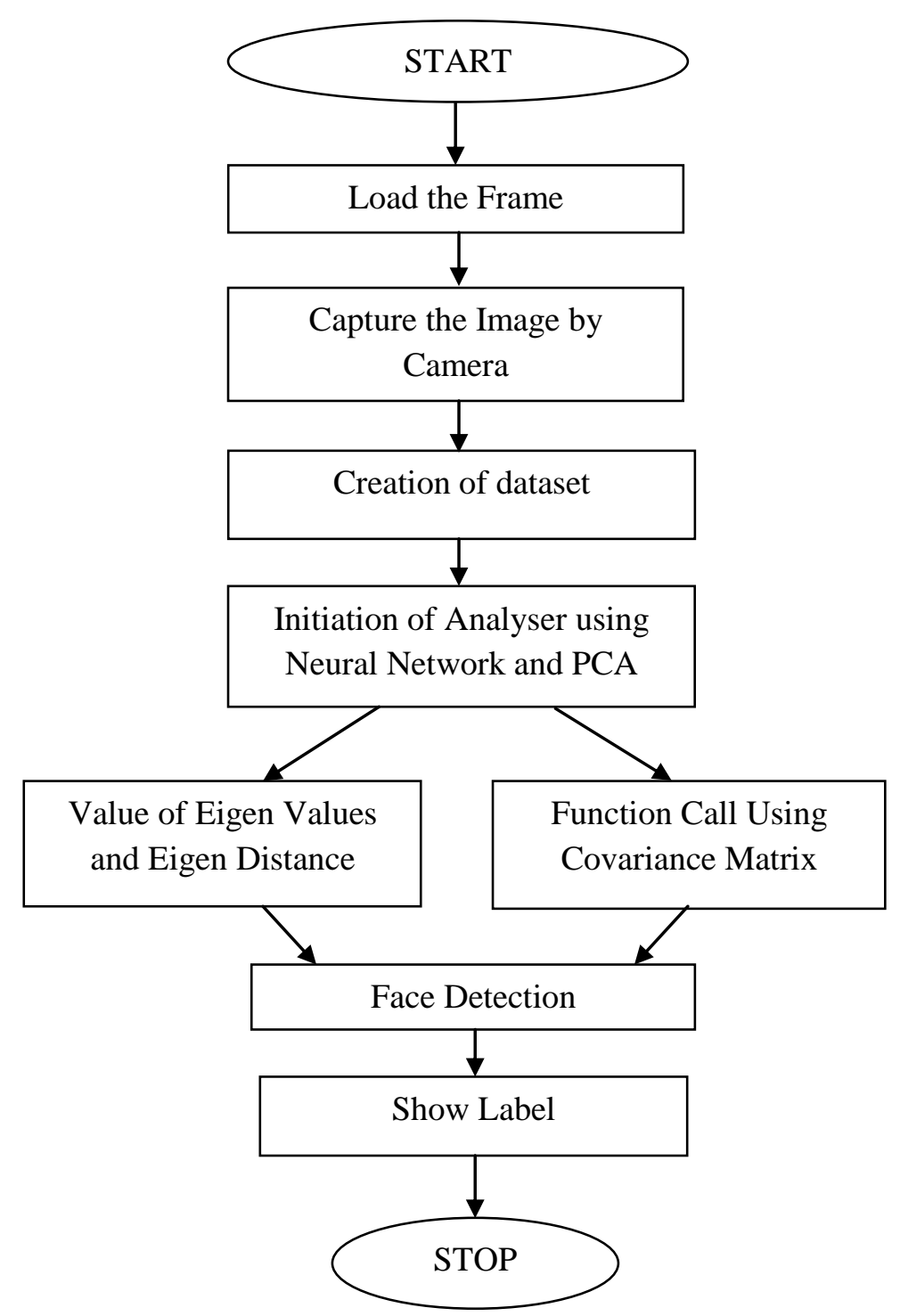

Figure 1. Proposed Methodology Using Neural Network

Goal of PCA is to reduce the dimensionality of the data by retaining as much as variation possible in our original data set. On the other hand dimensionality reduction implies information loss. In this paper, the

PCA performs several steps such:

Stage 1: Subtract the Mean of the data from each variable (our adjusted data)

Stage2: Calculate and form a covariance matrix. 


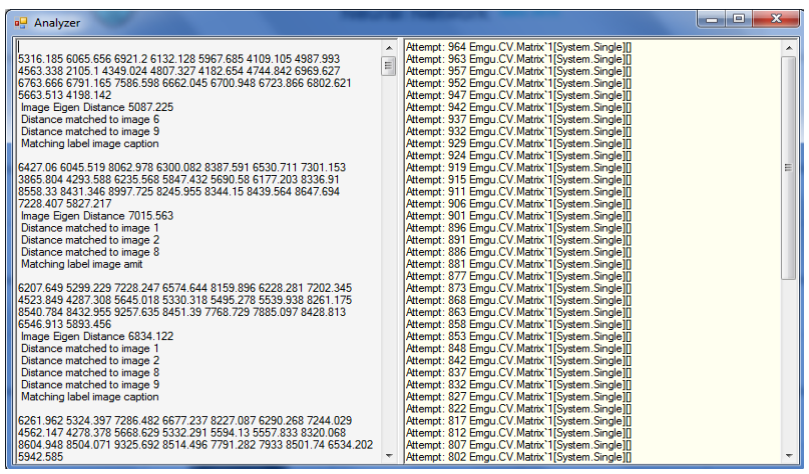

Figure 2. Covariance Matrix is Created [22]

Stage 3: Calculate Eigen vectors and Eigen values from the covariance matrix.

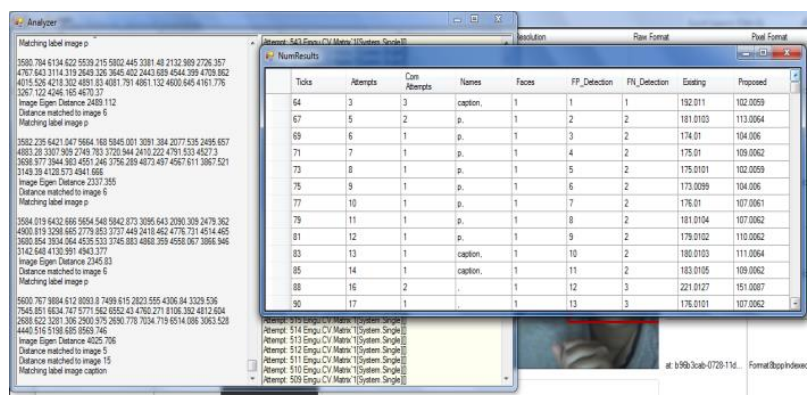

Figure 3. Eigen Result Analyzer [22]

Stage4: Choose a feature vector (a fancy name for a matrix of vectors)

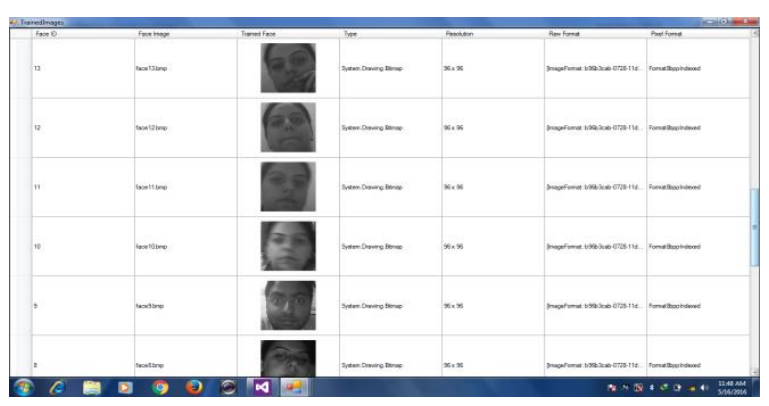

Figure 4. Data Set is Created [22]

Stage 5: Multiply the transposed feature vectors by the transposed adjusted data.

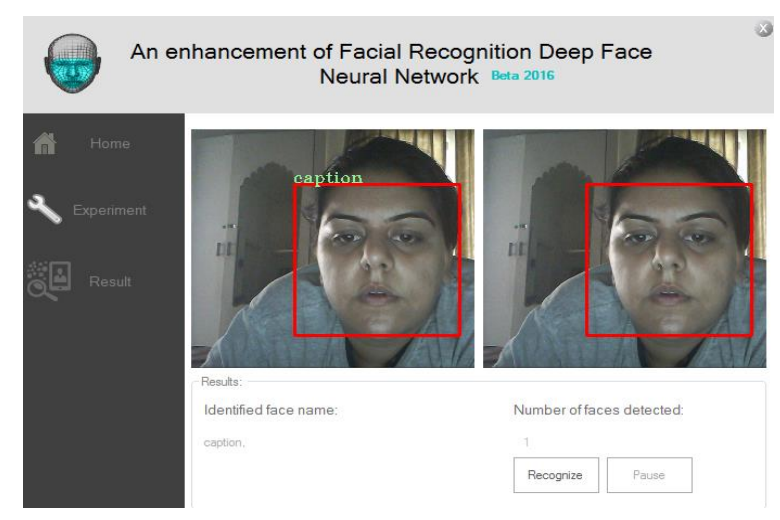

Figure 5. Recognition is Done 


\section{Flow for Face Recognition Process}

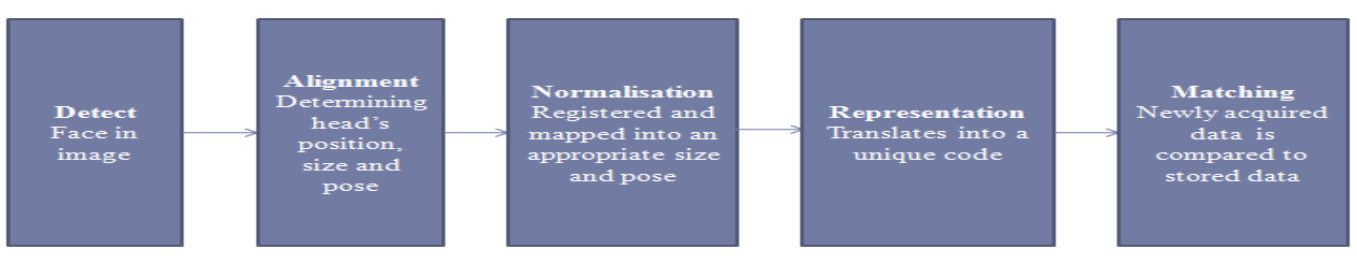

Figure 6. Flow Chart for Face Recognition

The Figure 6, depicts the flow of Face Recognition Process. Facial recognition methods suggest a few of steps that are capturing, analyzing and comparing your face to a database of stored images. The process used by the face recognition system to capture and compare images is described as below:

Step 1 Detection - Using the video camera, the recognition software searches the faces when the system is attached to a video surveillance system. $f$ there is a face in the view, it is detected within a fraction of a second.

Step 2 Alignment - Once the system has detected the face, and then it determines the head's position, size and pose. The system needs to be turned at least 35 degrees towards the camera to register the face.

Step 3 Normalization - The image of the head is scaled and rotated to register and map into an appropriate size and pose. Despite of the head's location and distance from the camera, normalization is performed. Light is not an issue in the normalization process.

Step 4 Representation - The system converts the facial data into a unique code after the normalization process. This coding process allows for comparison and easier representation of the newly acquired facial data to facial data which is already stored.

Step 5 Matching - The acquired (new) facial data is compared to the stored data and linked to at least one stored facial representation. Whether the features extracted from the newly acquired facial data are a match or not, is decided by the system. A match is declared if the score is above a predetermined threshold

\section{Approach}

The Eigen Face Recognizer class applies PCA on each image, the results of which will be an array of Eigen values that a Neural Network can be trained to recognize.

The method of which PCA is applied can vary at different stages so what will be demonstrated is a clear method for PCA application that can be followed. It is up for individuals to experiment in finding the best method for producing accurate results from PCA.

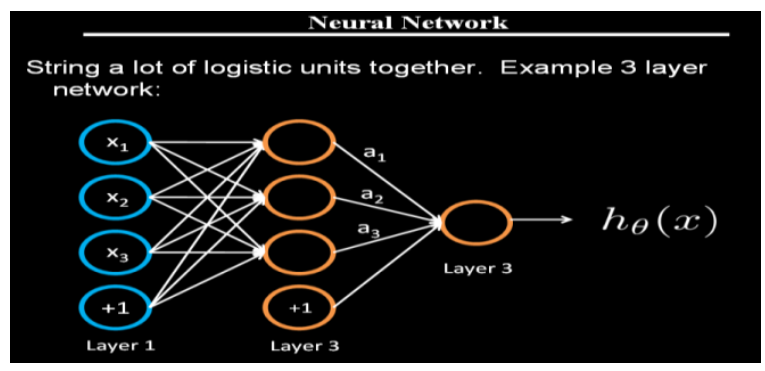

Figure 7(a). Working of Neural Network in First Stage 


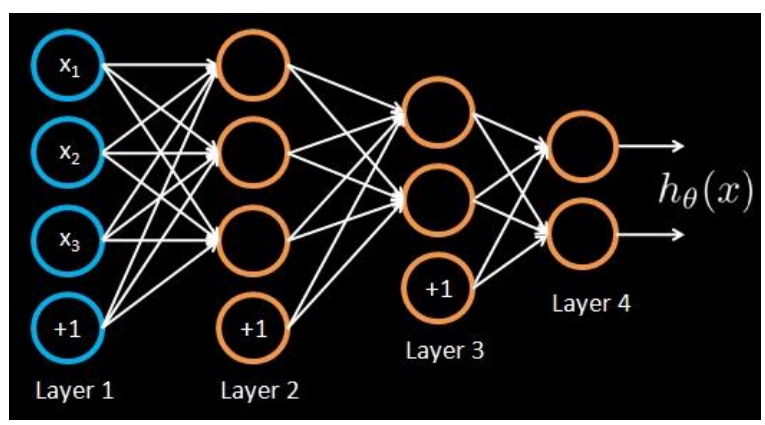

Figure 7(b). Working of Neural Network in Second Stage

\section{A. Covariance Matrix}

The basic Covariance equation for two dimensional data is:

$$
\operatorname{cov}(x, y)=\frac{\sum_{i-1}^{n}\left(x_{i}-\bar{x}\right)\left(y_{i}-\bar{y}\right)}{(n-1)}
$$

Which is similar to the formula for variance however, the change of $\mathrm{x}$ is in respect to the change in $\mathrm{y}$ rather than solely the change of $\mathrm{x}$ in respect to $\mathrm{x}$. In this equation $\mathrm{x}$ represents the pixel value and $\mathrm{x}$ is the mean of all $\mathrm{x}$ values, and on the total number of values.

\section{B. Eigen Values Vector}

Eigen values are a product of multiplying matrices however they are as special case. Eigen values are found by multiples of the covariance matrix by a vector in two dimensional space (i.e., a Eigenvector). This makes the covariance matrix the equivalent of a transformation matrix.

\section{Feature Vector}

Now a usually the results of Eigen values and Eigenvectors are not as clean as in the example above. In most cases the results provided are scaled to a length of 1 . Once Eigenvectors are found from the covariance matrix, the next step is to order them by Eigen value, highest to lowest. This gives you the components in order of significance. Here the data can be compressed and the weaker vectors are removed producing a lossy compression method, the data lost is deemed to be insignificant.

\section{Results and Discussion}

In this research paper the face recognition has focused on different techniques including the parameters such as time, false acceptance rate and cross correlation. These parameter increases the value of research work on the basis of previous work and the proposed work.

\subsection{Face Detection Time}

Face detection time defines to the psychological process by which human face locate and attend he faces in a visual scene and matches bit by bit from the database. At this stage, the symmetry measures the fitness value according to the time for verified the face. 
Table 1. Comparison of Images on the Basis of Time

\begin{tabular}{|l|l|l|l|l|}
\hline \multirow{4}{*}{ TIME } & Methodology & $\cdot$ & $\mathrm{p}$ & caption \\
\cline { 2 - 5 } & Previous & 183.01 & 179.01 & 194.01 \\
\cline { 2 - 5 } & Proposed & 101.05 & 111.01 & 111.06 \\
\hline
\end{tabular}

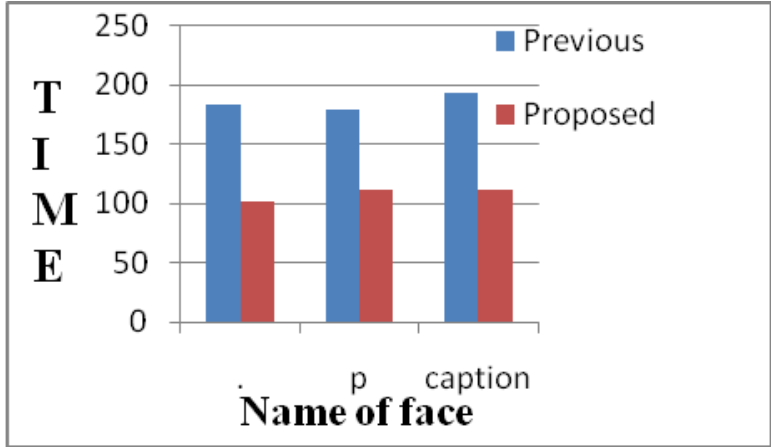

Figure 8. Bar Chart Representation of Comparative Analysis of TIME

From Table 1, and Figure 8, It is clearly shown that using proposed method the value of TIME is less than time required in previous method

\subsection{False Acceptance Rate}

The false acceptance rate, or FAR, is the measure of the likelihood that the biometric security system will incorrectly accept an access attempt by an unauthorized user. A system's FAR typically is stated as the ratio of the number of false acceptances divided by the number of identification attempts. Table 2, shows the FAR of different images.

Table 2. Comparison of Images on the Basis of FAR

\begin{tabular}{|l|l|l|l|l|}
\hline \multirow{4}{*}{ FAR } & Methodology & $\cdot$ & $\mathrm{p}$ & caption \\
\cline { 2 - 5 } & Previous & 0.568 & 0.588 & 0.238 \\
\cline { 2 - 5 } & Proposed & 0.29 & 0 & 0 \\
\hline
\end{tabular}

Figure 9, we have shown the comparative analysis of FAR for various images

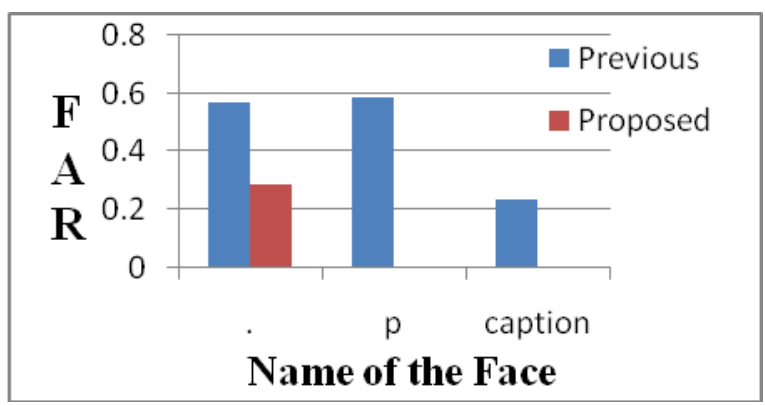

Figure 9. Shows the Comparative Analysis of FAR in the Form of Bar Chart Representation. It is Clearly Shown That Using Proposed Method the Value of FAR is Less Than Required in Previous Method 


\section{Conclusion}

Rather than focus on improving the performance for slower processors this program is designed to increase the performance on modern machines. It is often a desired, although impractical, to have real time image processing. Real time processing is closely linked to the accuracy of an image-processing algorithm. Faster algorithms are a result of processing less data and their ability to determine true from false data is inherently flawed. In video acquisition 30 frame per-second is deemed as standard, it's faster than what our eyes can cope with and thus provided smooth movement. In real world applications this is to slow for a computer to be accurate.

The proposed techniques are accessible for security applications which are created by specialists as a rule handle and may be support a large impacts adjusted; thus these research work have restricted abilities with spotlight on some all-around organized application. The proposed methodology is more successful and provides the better optimality in face recognition pattern. The process result will shows the better detection time as compared to previous research work.

\section{References}

[1] Y. Taigman, M. Yang, M. A. Ranzato and L. Wolf, "DeepFace: closing the gap to human level performance", (CVPR), IEEE Conference, (2014) June 23-28, pp. 1701-1708.

[2] C. Liu, "Capitalize on dimensionally increasing the techniques for improving face recognition grand challenge performance", TPAMI, vol. 11, no. 4, (2002), pp. 467-476.

[3] L. Shen, L. Bai and M. Fairhurst, "Bayesian Network wavelets and general discriminant analysis for face identification and verification", vol. 25, no. 5, (2007), pp. 553-563.

[4] V. 'Struc and N. Pave`si'c, "Gabor-based kernel partial-least-squares discrimination features for face recognition", Informatica, vol. 20, no. 1, (2009), pp. 115-138.

[5] M. Lades, J. Vorbruggen, J. Buhmann, J. Lange, C. V. D. Malsburg, R. Wurtz and W. Konen, "Distortion invariant object recognition in the dynamic link architectrue", IEEE Transactions on Computers, vol. 42, no. 3, (1993), pp. 300-311.

[6] L. Wang, Y. Li, C. Wang and H. Zhang, "2d Gabor face representation method for face recognition with ensemble and multichannel model", Image and Vision Computing, vol. 26, no. 6, (2008), pp. 820-828.

[7] K. Messer, J. Matas, J. Kittler, J. Luettin and G. Maitre, "Xm2vtsdb: the extended m2vts database", in Proceedings of AVBPA'99, Washington D.C., USA, (1999) March, pp. 72-77.

[8] A. Verma and M. Khera, "Comparative Study on Biometrics: a review", International Journal of Advanced Research in Computer Science and Software Engineering, vol. 4, no. 5, (2014), pp. 233-236.

[9] Y. Bakhshi, S. Kaur and P. Verma, "A Study based on Various Face Recognition Algorithms", International Journal of Computer Applications, vol 129, no. 13, (2015), pp 16-20.

[10] J. Harguess and J. K. Aggarwal, "A case for the average-half-face in 2D and 3D for face recognition", In Computer Vision and Pattern Recognition Workshops, 2009. CVPR Workshops 2009. IEEE Computer Society Conference onIEEE, (2009), pp. 7-12.

[11] A. K. Jain, B. Klare and U. Park, "Face recognition: Some challenges in forensics", In Automatic Face \& Gesture Recognition and Workshops (FG 2011), 2011 IEEE International Conference on, pp. 726733.

[12] J. Shermina, "Illumination invariant face recognition using discrete cosine transform and principal component analysis", In Emerging Trends in Electrical and Computer Technology (ICETECT), 2011 International Conference onIEEE, (2011), pp. 826-830.

[13] M. A. Lone, S. M. Zakariya and R. Ali, "Automatic Face Recognition System by Combining Four Individual Algorithms", In Computational Intelligence and Communication Networks (CICN), 2011 International Conference onIEEE, (2011), pp. 222-226.

[14] B. F. Klare, M. J. Burge, J. C. Klontz, R. W. V. Bruegge and A. K. Jain, "Face recognition performance: Role of demographic information", Information Forensics and Security, IEEE Transactions on, vol. 7, no. 6, (2012), pp. 1789-1801.

[15] G. P. Teja and S. Ravi, "Face recognition using subspaces techniques", In Recent Trends In Information Technology (ICRTIT), 2012 International Conference onIEEE, (2012), pp. 103-107.

[16] T. Horiuchi and T. Hada, "A complementary study for the evaluation of face recognition technology", In Security Technology (ICCST), 2013 47th International Carnahan Conference onIEEE, (2013), pp. 1-5.

[17] K. Niinuma, H. Han and A. K. Jain, "Automatic multi-view face recognition via 3D model based pose regularization", In Biometrics: Theory, Applications and Systems (BTAS), 2013 IEEE Sixth International Conference on. IEEE, (2013), pp. 1-8. 
[18] B. Cai, S. Xiao, L. Jiang, Y. Wang and X. Zheng, "A rapid face recognition BCI system using singletrial ERP”, In Neural Engineering (NER), 2013 6th International IEEE/EMBS Conference onIEEE, (2013), pp. 89-92.

[19] G. Mahalingam, K. Ricanek and A. Midori, "Investigating the Periocular-Based Face Recognition across Gender Transformation", (2014).

[20] M. Kafai, L. An and B. Bhanu, "Reference Face Graph for Face Recognition", Oravec, Milos. "Feature extraction and classification by machine learning methods for biometric recognition of face and iris." In ELMAR (ELMAR), 2014 56th International SymposiumIEEE, (2014), pp. 1-4.

[21] G. Kaur, S. Kaur and A. Walia."Face recognition using PCA Deep Face Method "In International Journal of computer science and mobile computing, vol. 5, (2016) May, pp. 359-366.

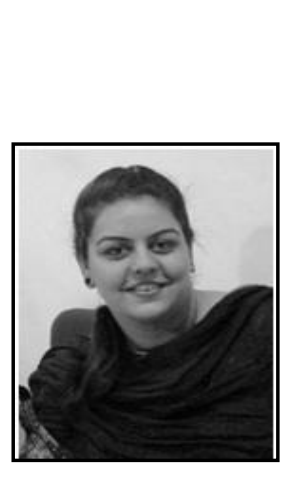

\begin{abstract}
Author
Gurpreet Kaur, I am working as an Assistant Professor in CT Group of Institutions Shahpur, Jalandhar, India and currently pursuing M.Tech in the Department of Computer Science Engineering at CT Institute of Engineering Management \& Technology, Jalandhar, India and have received B. Tech in Computer Science Engineering from Lovely Professional University. My area of interest is in Digital Image Processing.
\end{abstract}

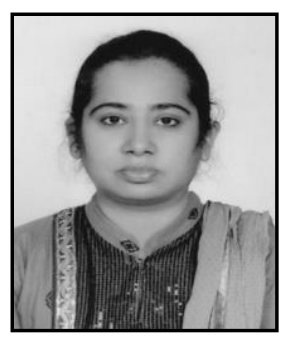

Sukhvir Kaur, I am working as Assistant Professor in CTIEMT, Jalndhar, India. I have 10 years of teaching experience. I have done my M.Tech (CSE) from DAVIET, Jalandhar, Punjab, India and B Tech (Computer Science Engineering) from BBSEC, Fatehgarh Sahib, under Punjab Technical University Jalandhar INDIA. .My area of interest is Digital Image Processing. 
International Journal of Signal Processing, Image Processing and Pattern Recognition Vol. 9, No. 8 (2016) 